\title{
Fingolimod Treatment in Relapsing-Remitting Multiple Sclerosis Patients: A Prospective Observational Multicenter Postmarketing Study
}

\author{
Rocco Totaro, ${ }^{1}$ Caterina Di Carmine, ${ }^{1}$ Gianfranco Costantino, ${ }^{2}$ \\ Roberta Fantozzi, ${ }^{3}$ Paolo Bellantonio, ${ }^{3}$ Aurora Fuiani, ${ }^{2}$ Ciro Mundi, \\ Stefano Ruggieri, ${ }^{3}$ Carmine Marini, ${ }^{1}$ and Antonio Carolei ${ }^{1}$ \\ ${ }^{1}$ Department of Neurology, University of L'Aquila, L'Aquila, Italy \\ ${ }^{2}$ Department of Neurology, Ospedali Riuniti, Foggia, Italy \\ ${ }^{3}$ Department of Neurology, IRCCS MEUROMED, Pozzilli, Italy \\ Correspondence should be addressed to Rocco Totaro; rocco.totaro@univaq.it
}

Received 29 January 2015; Revised 10 June 2015; Accepted 14 June 2015

Academic Editor: Bianca Weinstock-Guttman

Copyright (C) 2015 Rocco Totaro et al. This is an open access article distributed under the Creative Commons Attribution License, which permits unrestricted use, distribution, and reproduction in any medium, provided the original work is properly cited.

Objective. The aim of this prospective observational multicenter postmarketing study was to evaluate fingolimod efficacy in a real world clinical setting. Methods. One hundred forty-two subjects with relapsing-remitting multiple sclerosis (RRMS) were enrolled in three multiple sclerosis centers throughout Central and Southern Italy between January 2011 and September 2013. After enrollment, regular visits and EDSS assessment were scheduled every 3 months, and MRI scan was obtained every 12 months. Patients were followed up from 1 to 33 months (mean $14.95 \pm 9.15$ months). The main efficacy endpoints included the proportion of patients free from clinical relapses, from disability progression, from magnetic resonance imaging activity, and from any disease activity. Results. Out of 142 patients enrolled in the study, $88.1 \%$ were free from clinical relapse and $69.0 \%$ were free from disability progression; $68.5 \%$ of patients remained free from new or newly enlarging T2 lesions and 81.7\% of patients were free from gadolinium enhancing lesions. Overall the proportion of patients free from any disease activity was $41.9 \%$. Conclusions. Our data in a real world cohort are consistent with previous findings that yield convincing evidence for the efficacy of fingolimod in patients with RRMS.

\section{Introduction}

Multiple sclerosis is a chronic inflammatory disease in which autoreactive lymphocytes induce inflammatory damage to myelin sheaths of the central nervous system (CNS). The morphologic hallmarks of multiple sclerosis are demyelination, inflammation, axonal damage, and gliosis with loss of oligodendrocytes and neurons. Treatment options have widened in the last decade, leading to fingolimod (FTY270) approval as the first oral agent proved to be effective in reducing relapse rate and disability progression in relapsing remitting multiple sclerosis (RRMS). Fingolimod acts as a sphingosine-1-phosphate receptor (S1PR) modulator retaining pathogenic autoreactive $\mathrm{T}$ cells in the lymph nodes and preventing their infiltration into the CNS [1-4]. In addition, some studies suggest that fingolimod biological activity would be underpinned, to some extent, by its direct interaction with neural cells expressing S1PR $[5,6]$.

The efficacy of oral fingolimod was investigated in two large phase 3 clinical trials comparing oral fingolimod doses of $0.5 \mathrm{mg}$ and $1.25 \mathrm{mg}$ once daily in RRMS [7-10]. The FTY720 Research Evaluating Effects of Daily Oral Therapy in Multiple Sclerosis (FREEDOMS) trial showed significant efficacy of fingolimod in reducing the annualized relapse rate (ARR), the risk of disability progression, and the number of new or newly enlarging brain lesions on magnetic resonance imaging (MRI) $[7,8,10]$. The second pivotal trial (Trial Assessing Injectable Interferon versus FTY720 Oral in RelapsingRemitting Multiple Sclerosis, TRANSFORMS) confirmed preliminary results, as fingolimod proved to be effective in reducing the ARR and MRI disease activity measures but 
TABLE 1: Patient demographics and baseline clinical characteristics.

\begin{tabular}{|c|c|c|c|c|}
\hline & Total $(n=142)$ & Men $(n=47)$ & Women $(n=95)$ & $p$ value \\
\hline Age $(y)$ & $39.56 \pm 9.11$ & $39.63 \pm 8.15$ & $39.53 \pm 9.59$ & 0.954 \\
\hline Duration of disease (y) & $11.37 \pm 6.74$ & $11.01 \pm 6.13$ & $10.62 \pm 7.44$ & 0.759 \\
\hline Number of previous DMT & $1.51 \pm 0.83$ & $1.47 \pm 0.80$ & $1.54 \pm 0.85$ & 0.644 \\
\hline Duration of DMT (months) & $59.52 \pm 45.49$ & $32.02 \pm 46.45$ & $58.28 \pm 45.21$ & 0.647 \\
\hline Previous immunosuppressant therapy & $19.01 \%$ & $25.53 \%$ & $15.78 \%$ & 0.164 \\
\hline Previous use of natalizumab & $19.71 \%$ & $12.76 \%$ & $23.15 \%$ & 0.143 \\
\hline \multicolumn{5}{|c|}{ In the year prior to commencing fingolimod } \\
\hline EDSS score & $2.52 \pm 1.02$ & $2.36 \pm 1.02$ & $2.60 \pm 1.08$ & 0.222 \\
\hline ARR & $1.14 \pm 0.71$ & $1.06 \pm 0.60$ & $1.18 \pm 0.78$ & 0.373 \\
\hline New or newly enlarging T2 lesions & $72.3 \%$ & $70.2 \%$ & $73.4 \%$ & 0.690 \\
\hline Gadolinium enhancing lesions & $49.6 \%$ & $44.7 \%$ & $52.1 \%$ & 0.404 \\
\hline
\end{tabular}

Data are mean \pm SD unless otherwise indicated.

ARR: annualized relapse rate.

DMT: disease modifying therapy.

EDSS: Expanded Disability Status Scale.

failed to slow disability progression, compared to interferon beta 1-a [9].

In phase 2, phase 3, and extension studies, fingolimod exhibited a relatively good safety and tolerability profile. Most commonly reported adverse events in the fingolimod group were mild infections, mainly of the lower respiratory tract, increased alanine aminotransferase levels, bradycardia, first and second degree atrioventricular blocks at the time of first administration, hypertension, macular edema, mild decrease in lung function, and lowering of peripheral blood lymphocyte count, as expected from the mechanism of action of fingolimod [11-13].

The European Medicines Agency (EMA) approved fingolimod as a single agent disease modifying therapy in patients with unsatisfactory disease control despite treatment with a beta interferon or in treatment-naïve patients with rapidly evolving severe multiple sclerosis, providing a second-line treatment option in highly active RRMS. A recent observational population study showed similar efficacy of fingolimod compared with natalizumab on clinical measures, as defined by ARR and risk of disability progression, in an unselected RRMS cohort [14].

The aim of this prospective postmarketing multicenter observational study was to evaluate the clinical efficacy of fingolimod in RRMS patients in a real clinical practice setting.

\section{Materials and Methods}

2.1. Patients and Clinical Parameters. We report prospectively collected data from 142 patients diagnosed with RRMS according to McDonald criteria, in three multiple sclerosis centers throughout Central and Southern Italy, starting fingolimod treatment. Patients were consecutively enrolled between January 2011 and September 2013.

Baseline demographic and clinical data are reported in Table 1. Out of 142 patients, 95 (66.9\%) were female; mean age was $39.56 \pm 9.11$ years $(39.63 \pm 8.15$-year males, $39.53 \pm$ 9.59-year females) with a mean disease duration of $11.37 \pm$ 6.74 years $(11.01 \pm 6.13$-year males, $10.62 \pm 7.44$-year females). Demographic characteristics did not differ significantly between groups.

Mean duration of the fingolimod treatment was $14.95 \pm$ 9.15 months (range 1-33 months).

Thirty-four patients (23.94\%) received fingolimod $\geq 24$ months, 47 patients (33.09\%) received fingolimod between 12 and 23 months, 33 patients $(23.23 \%)$ received fingolimod between 6 and 11 months, and 28 patients (19.71\%) received fingolimod between 1 and 5 months.

Patients were included in accordance with the Italian Drug Agency (AIFA) eligibility criteria for reimbursement of the drug. Criteria included patients on previous immunomodulant treatment for at least 12 months who had experienced at least one relapse in the last year with at least $9 \mathrm{~T} 2$ lesions at MRI or an increased lesion burden or at least one gadolinium-enhanced lesion or patients with severe multiple sclerosis with a fast progression/evolution, even if not previously treated with immunomodulant treatments, with $\geq 2$ relapses with accumulation of disability during the last year, and with new T2- or gadolinium-enhanced lesions on MRI, compared with a previous MRI examination conducted during the previous 12 months.

Patients switching from natalizumab to fingolimod were also recruited and started treatment after a wash-out period of three months. All these patients discontinued natalizumab due to safety concerns for positive JCV status and overall treatment duration longer than 24 months, despite being clinically and radiologically stable under natalizumab treatment.

Patients underwent complete cardiac evaluation before starting fingolimod in order to exclude heart rate and atrioventricular conduction alterations. Moreover, a continuous electrocardiographic monitoring for six hours after the administration of the first dose was performed in all patients. Every hour, arterial pressure values were also registered.

Ophthalmologic evaluation with optical coherence tomography was performed at baseline, at 3 and 6 months and every 6 months thereafter, in order to monitor potential adverse events such as macular oedema. 
Neurological examination and EDSS assessment were performed at baseline and during follow-up visits. EDSS was registered at baseline and every 6 months.

MRI scans were obtained at baseline, 12 and 24 months. Imaging data were collected at MRI facilities of the participating sites and reviewed by experienced neuroradiologists. MRI imaging was performed at $1.5 \mathrm{~T}$ and at least axial FLAIR, T2 weighted, and T1 gadolinium-enhanced sequences were obtained for all exams. MRI scans were analyzed for new or newly enlarging T2 lesions and gadolinium enhancing lesions.

Adverse events were recorded during the routine clinic visits or at their occurrence.

All patients were treated at a dose of fingolimod $0.5 \mathrm{mg}$ once daily.

The study was approved by the Azienda Sanitaria Locale Avezzano-Sulmona-L'Aquila Ethics Committee and conducted in conformance with the ethical principles of the Declaration of Helsinki. Written informed consent was obtained from all patients.

2.2. Study Endpoints. The main end points were the proportion of patients free from clinical relapses; from confirmed disability progression, as defined by sustained increase of $\geq 1.0$ point in the EDSS score (or $\geq 1.5$ points if the baseline was $<1.0)$ confirmed after 12 weeks; from new or newly enlarging T2 lesions; from gadolinium enhancing lesions; from any disease activity, as defined by the occurrence of relapse or confirmed disease progression or new disease activity at MRI.

ARR and EDSS score variations at 6, 12, 18, and 24 months with respect to basal values were also considered.

An intention to treat analysis was planned.

2.3. Statistical Analysis. Continuous variables were reported as means, standard deviations and range while discrete data were reported in contingency tables as absolute and relative frequencies.

The Mann-Whitney $U$-test or Wilcoxon tests were used where appropriate. The Cox multivariate model was used to test the influence of prognostic factors (age, sex, duration of the disease, number of previous treatments, EDSS score before fingolimod start, number of contrast-enhancing lesions at MRI before fingolimod start, previous immunosuppressant treatments, and previous natalizumab treatment).

The selected critical value for statistical significance was 0.05 . Contingency tables were analyzed by $\chi^{2}$ test where appropriate. Binary efficacy responses (yes/no, present/ absent) were reported as absolute and relative frequencies. Furthermore, the appearance of events during the study was performed by the Kaplan-Meier (product-limit) survival analysis.

\section{Results}

In the overall population, the cumulative proportion of patients free from clinical relapse was $88.1 \%$ (Figure 1). Figure 2 shows the cumulative proportion of patients free

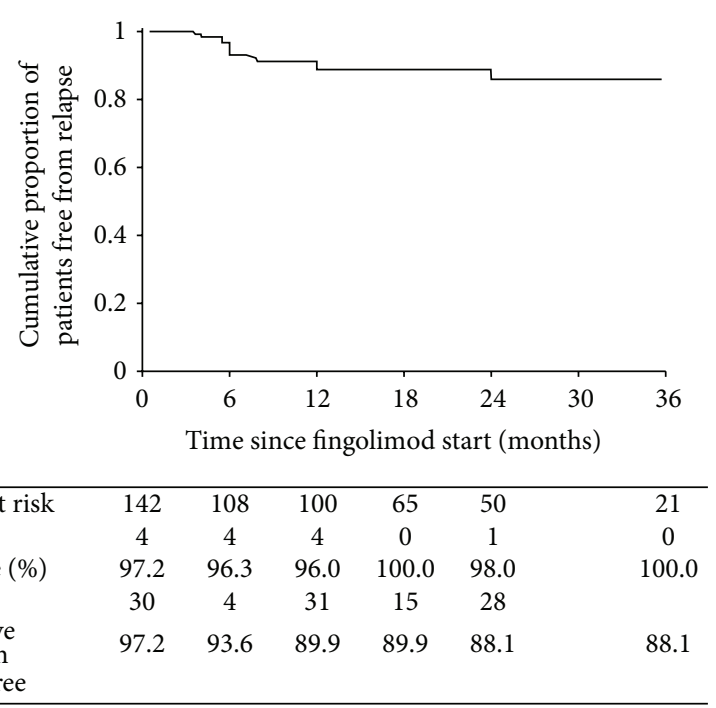

FIGURE 1: Kaplan-Meyer plot of cumulative proportion of patients remaining free from relapse over time from start of fingolimod therapy.

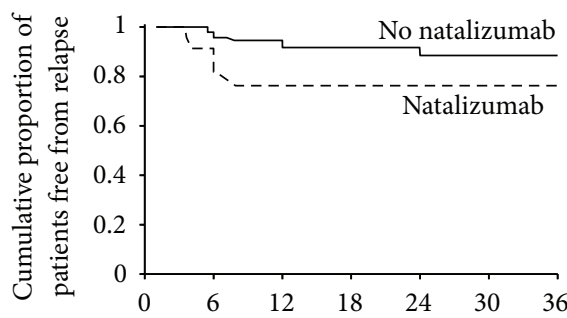

Time since fingolimod start (months)

\begin{tabular}{|c|c|c|c|c|c|c|c|}
\hline \multirow{5}{*}{ 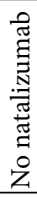 } & Number at risk & 114 & 89 & 84 & 56 & 45 & 20 \\
\hline & No event & 2 & 2 & 3 & 0 & 1 & 0 \\
\hline & Event-free (\%) & 98.2 & 97.8 & 96.4 & 100.0 & 97.8 & 100.0 \\
\hline & Censored & 23 & 3 & 25 & 11 & 24 & \\
\hline & $\begin{array}{l}\text { Cumulative proportion } \\
\text { of event-free }\end{array}$ & 98.2 & 96.0 & 92.5 & 92.5 & 90.5 & 90.5 \\
\hline \multirow{5}{*}{ 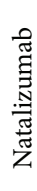 } & Number at risk & 28 & 19 & 16 & 9 & 5 & 1 \\
\hline & No event & 2 & 2 & 1 & 0 & 0 & 0 \\
\hline & Event-free (\%) & 92.9 & 89.5 & 93.8 & 100.0 & 100.0 & 100.0 \\
\hline & Censored & 7 & 1 & 6 & 4 & 4 & \\
\hline & $\begin{array}{l}\text { Cumulative proportion } \\
\text { of event-free }\end{array}$ & 92.9 & 83.1 & 77.9 & 77.9 & 77.9 & 77.9 \\
\hline
\end{tabular}

FIGURE 2: Kaplan-Meyer plot of cumulative proportion of patients remaining free from relapse by prior exposure to natalizumab over time from start of fingolimod therapy.

from clinical relapse with respect to prior exposure to natalizumab. Patients without previous natalizumab exposure had improved relapse-free survival compared with those previously treated with natalizumab $(90.5 \%$ versus $77.9 \%$; $p=$ $0.021)$.

Mean time to relapse was 29.6 months (95\% confidence interval [CI], 28.025-31.202). Time to first relapse significantly decreased in patients receiving previous natalizumab treatment $(23.767 \pm 2.218$ versus $30.411 \pm 0.778$; $p=0.021)$.

ARR significantly reduced after treatment with fingolimod. Values decreased from 1.14 to 0.14 at 6 months, to 


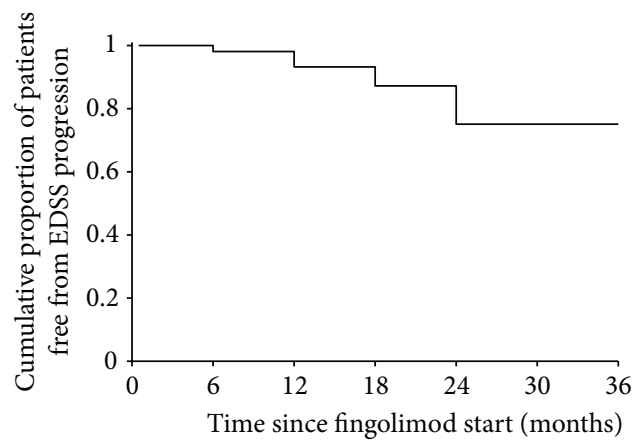

Number at risk

No event

Event-free (\%)

Censored

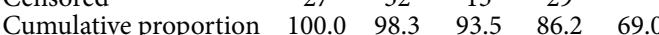

of event-free

FIGURE 3: Kaplan-Meyer plot of cumulative proportion of patients remaining free from Expanded Disability Status Scale (EDSS) progression over time from start of fingolimod therapy.

0.11 at one year, and to 0.09 at 2 years of treatment $(p<$ 0.0001). At 6 months of follow-up, ARR was significantly associated with the number of relapses in the year preceding the start of fingolimod treatment $(p=0.049)$ and with the previous use of natalizumab $(p=0.018)$. No associations were found at 12 and 24 months.

As shown in Figure 3, the cumulative proportion of patients free from confirmed EDSS progression was $69.0 \%$. During the study period, mean EDSS score decreased from $2.7 \pm 1.1$ at baseline to $2.5 \pm 1.1$ at 18 months $(p=0.13$ ). EDSS evaluation at 24 months disclosed a trend toward further decrease, though sample size was too small to reach statistical significance $(2.7 \pm 1.1$ versus $2.3 \pm 0.9 ; p=$ 0.501). Mean time to confirmed EDSS progression was 28.6 months (95\% CI, 26.9-30.3). Subgroup analysis failed to show any difference in time to disability progression for patients undergoing previous natalizumab treatment. Multivariate regression analysis showed no correlation between the risk of disability progression and age, gender, disease duration, number of previous treatments, previous immunosuppressant therapies, EDSS at baseline, previous contrast enhancing lesions, or previous natalizumab treatment.

MRI scans were obtained at baseline in all patients and in all 81 and 34 patients that reached 12 and 24 months of followup, respectively.

As shown in Figure 4, 68.5\% of patients were free from new or newly enlarging T2 lesions. Time to recurrence of new T2 lesions was 25.8 months, on average (95\% CI, 23.8-27.9). Multivariate regression analysis found that EDSS score at baseline was the only predictor of new or newly enlarging $\mathrm{T} 2$ lesion occurrence, as EDSS score at baseline was significantly lower in patients with new T2 lesions on follow-up MRI ( $p=$ $0.016)$.

Figure 5 shows that $81.7 \%$ of patients were free from new gadolinium enhancing lesions. Mean time to the appearance of a new enhancing lesion was 28.9 months (95\% CI, 27.230.7). New contrast enhancing lesions were more common in

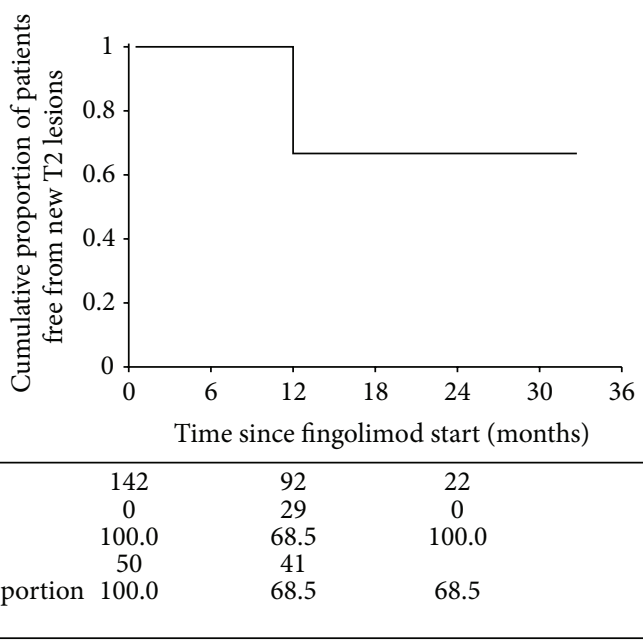

FIGURE 4: Kaplan-Meyer plot of cumulative proportion of patients remaining free from new or newly enlarging T2 lesions over time from start of fingolimod therapy.

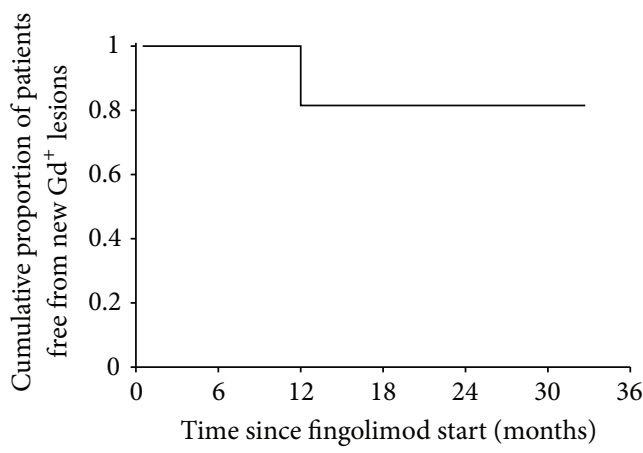

\begin{tabular}{lccc}
\hline Number at risk & 87 & 82 & 13 \\
No event & 0 & 15 & 0 \\
Event-free $(\%)$ & 100.0 & 81.7 & 100.0 \\
Censored & 6 & 69 & \\
$\begin{array}{l}\text { Cumulative proportion } \\
\text { of event-free }\end{array}$ & 100.0 & 81.7 & 81.7 \\
\hline
\end{tabular}

FIGURE 5: Kaplan-Meyer plot of cumulative proportion of patients remaining free from new gadolinium enhancing $\left(\mathrm{Gd}^{+}\right)$lesions at magnetic resonance imaging over time from start of fingolimod therapy.

patients undergoing previous natalizumab treatment $(26.7 \%$ versus $15.3 \%, p=0.046$ ).

As shown in Figure 6, the cumulative proportion of patients free from any disease activity was $41.9 \%$. Multivariate regression analysis revealed lower EDSS score at baseline as the only predictor of disease activity recurrence after fingolimod start $(p=0.018)$.

All patients were discharged at the end of the 6 hours of monitoring, but 8 patients (5.6\%) needed 2 hours of extended monitoring due to asymptomatic bradycardia. All these 8 patients were discharged after the extended monitoring. No significant modification of arterial pressure values was also registered. 


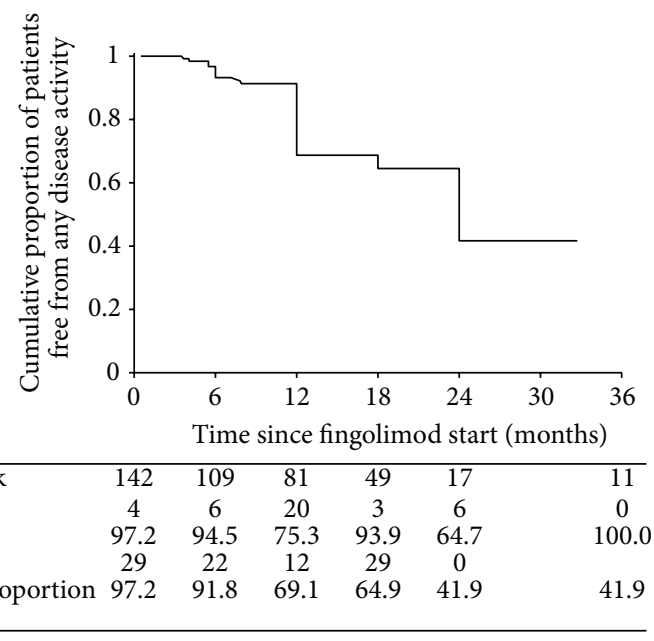

FIgURE 6: Kaplan-Meyer plot of cumulative proportion of patients remaining free from any disease activity over time from start of fingolimod therapy.

During the follow-up no serious adverse events were reported. However, headache was reported by 25 patients (17.6\%), diarrhea by 7 patients (4.9), and arterial hypertension by 3 patients $(2.1 \%)$.

Therapy was stopped in 13 patients (9.1\%). In 7 patients (4.9\%) the interruption was due to lack of efficacy, in 2 patients $(1.4 \%)$ it was due to general intolerance, and in 3 patients $(2.1 \%)$ it was stopped due to the patient's decision, and one patient $(0.7 \%)$ was lost to follow-up.

\section{Discussion}

In this prospective multicenter clinical practice study, fingolimod treatment was effective in providing freedom from disease activity and preventing clinical relapse in patients with RRMS. After starting fingolimod, the cumulative proportion of patients free from clinical relapse throughout the follow-up was $88.1 \%$, and $69.0 \%$ of patients remained free from disability progression. Disability progression rates curves disclosed a trend toward further improvement over time. Cumulatively during the study period, almost half of patients were free from any disease activity, that is, no relapses, no disability progression, no new or enlarged T2 lesions, and no gadolinium enhancing lesions.

Of interest, relapse rates were higher in patients with prior natalizumab treatment compared with patients without previous natalizumab exposure (relapse-free survival $77.9 \%$ versus $90.5 \%$, resp.). Although prior receipt of natalizumab was associated with a shorter time to first relapse, there was no difference in time to disability progression between patients who had or had not undergone prior natalizumab treatment. It is a widely held notion that natalizumab withdrawal is associated with possible severe disease reactivation occurring within the first three or up to seven months after natalizumab discontinuation. Rather than rebound activity, early relapses are likely to be underpinned by the clearance of natalizumab biological effect prompting recurrence of disease activity.
Hence, higher relapse rate and new gadolinium enhancing lesions at 6 months after switching from natalizumab to fingolimod could be possibly driven by early disease recrudescence of disease activity. Recent studies suggest that a washout period as short as 4 to 4 weeks should be preferable in order to prevent severe disease reactivation after switching from natalizumab to fingolimod [15]. Nevertheless, our population underwent a three-month wash-out period as required by regulatory agencies in order to prevent potential safety issues from overlapping immunomodulatory effects.

While no direct comparisons are intended, it is of interest to review the findings observed in phase 3 clinical trials of fingolimod and natalizumab in patients with RRMS. The pivotal phase 3 clinical trials, FREEDOMS [7] and TRANSFORMS [9], each of which enrolled over 1000 patients, showed that fingolimod at doses of $0.5 \mathrm{mg}$ and $1.25 \mathrm{mg}$ once daily significantly reduced ARR and MRI disease activity compared with placebo (FREEDOMS) and interferon betala (TRANSFORMS) in patients with RRMS. In the 24month FREEDOMS study, fingolimod also slowed disability progression, whereas no significant reductions in disability progression had emerged during the 12 months of the TRANSFORMS study. During the 24-month period of the FREEDOMS study, fingolimod reduced the risk of disability progression confirmed at three months (17.7\% fingolimod $0.5 \mathrm{mg} ; 16.6 \%$ fingolimod $1.25 \mathrm{mg} ; 24.1 \%$ placebo) [7]. Both fingolimod doses also showed a reduction in the aggregate annualized relapse rate $(0.18$ fingolimod $0.5 \mathrm{mg}, 0.16 \mathrm{fin}-$ golimod $1.25 \mathrm{mg}$ ) versus placebo (0.40), representing relative reductions in annualized relapse rate of $54 \%$ and $60 \%$, respectively.

In the TRANSFORMS study the annualized relapse rate was 0.16 in the $0.5 \mathrm{mg}$ fingolimod group and $0.20 \mathrm{mg}$ in the $1.25 \mathrm{mg}$ fingolimod group versus 0.33 in the interferon group [9].

In the pivotal AFFIRM phase 3 trial of intravenous natalizumab, the 2 -year relapse-free rate was $72.41 \%$ in the natalizumab group, compared with $46.35 \%$ with placebo [16]. In a clinical practice setting, results from 343 consecutive patients with RRMS who started treatment with natalizumab at 12 Italian multiple sclerosis centers have recently been reported [17]. Over 42 months of follow-up, the cumulative proportion of patients free from relapse was $68 \%, 93 \%$ were free from EDSS progression, and $77 \%$ were free from MRI activity. Fifty-three percent were free from any disease activity. There was a reduction of ARR of approximately 90\% over the follow-up period, compared with the year preceding the start of natalizumab treatment.

Meanwhile, in a recent observational cohort study of 427 RRMS patients receiving either fingolimod or natalizumab, the risk of disability progression was lower in the fingolimod versus natalizumab group (87.39\% versus $82.7 \%$, resp.) and a greater proportion of patients receiving fingolimod was relapse-free at 12 months (75.79\% fingolimod versus $71.73 \%$ natalizumab) [14]. The relapse rate was substantially reduced in both treatment groups within three months of commencing treatment and remained at similar low levels in each group throughout follow-up. Similarly, EDSS was unchanged 
or improved in similar proportions of both groups and remained stable over 12 months of follow-up.

Treatment with fingolimod was well tolerated, and no patients had any severe adverse events. However, headache was the most common adverse event reported (17.6\%). During the study, no concerns with cardiac function were encountered, and no patients had clinically significant bradycardia.

The main focus of our study was to provide real world efficacy data from patients representative of the Italian relapsingremitting multiple sclerosis population meeting the eligibility criteria of the AIFA for treatment with fingolimod. Although direct comparisons cannot be made, our results in a real world clinical practice setting are to great extent in line with those observed in large clinical trials.

Our findings yield further information to the growing body of evidence for the use of fingolimod in multiple sclerosis in Europe. A recent review article summarized data on the efficacy and safety of fingolimod in patients eligible for therapy according to the European Union label to provide practical guidance on the use of fingolimod in the clinic [18]. In addition to pooled data from clinical trials, the review examined findings from recent European studies of realworld patient populations and concluded that, unlike some newer oral therapies for multiple sclerosis, fingolimod has a well-established safety and efficacy profile. Until additional long-term data on fingolimod are available, clinicians should implement appropriate routine patient monitoring measures to guide and optimize treatment benefits and to minimize adverse events.

We acknowledge that patients enrolled in our postmarketing study were quite heterogeneous in terms of followup length, ranging from 1 to 33 months; nevertheless mean follow-up was 14.95 months, and follow-up data were available for at least $57 \%$ and $23.9 \%$ of patients at 12 and 24 months, respectively. We decided to analyze data of all enrolled patients since literature data indicated an early action of fingolimod due to its mechanism of action [1-5]. Moreover, we included patients starting fingolimod after treatment with natalizumab who are at high risk of relapse in the first months of therapy. Thus, patients with few months of follow-up were also comparable.

We also acknowledge that data from patients with prior natalizumab treatment might have yielded mixed results in regard to previous disease activity and duration. Yet, the aim of our investigation was to perform a clinical study in real world setting as to provide efficacy data from a sample of patients representative of the overall population treated with fingolimod.

\section{Conclusions}

In our study, fingolimod positively influenced the course of RRMS in terms of degree of reduction of relapse, stabilization of EDSS, and reduction of MRI activity. Our data, showing no disease activity in about $50 \%$ of patients, confirmed the results of clinical trials.

\section{Abbreviations}

$\begin{array}{ll}\text { AIFA: } & \text { Italian Drug Agency } \\ \text { ARR: } & \text { Annualized relapse rate } \\ \text { CI: } & \text { Confidence interval } \\ \text { CNS: } & \text { Central nervous system } \\ \text { EDSS: } & \text { Expanded Disability Status Scale } \\ \text { EMA: } & \text { European Medicines Agency } \\ \text { FREEDOMS: } & \text { FTY720 Research Evaluating Effects of } \\ & \text { Daily Oral Therapy in Multiple Sclerosis } \\ \text { MRI: } & \text { Magnetic resonance imaging } \\ \text { MS: } & \text { Multiple sclerosis } \\ \text { RRMS: } & \text { Relapsing-remitting multiple sclerosis } \\ \text { S1PR: } & \text { Sphingosine-1-phosphate receptor } \\ \text { TRANSFORMS: } & \text { Trial Assessing Injectable Interferon } \\ & \text { versus FTY720 Oral in Relapsing- } \\ & \text { Remitting Multiple Sclerosis. }\end{array}$

\section{Conflict of Interests}

R. Totaro received funding for travel or speaker honoraria from Bayer Schering Pharma, Biogen Idec, Merck Serono, Novartis, Sanofi-Aventis, and TEVA. G. Costantino received funding for travel or speaker honoraria from Bayer Schering Pharma, Biogen Idec, Merck Serono, Novartis, SanofiAventis, and TEVA. P. Bellantonio received funding for travel or speaker honoraria from Bayer Schering Pharma, Biogen Idec, Merck Serono, Novartis, Sanofi-Aventis, and TEVA.

\section{Authors' Contribution}

Rocco Totaro, Gianfranco Costantino, and Paolo Bellantonio contributed to conception and design of the paper. Rocco Totaro contributed to study coordination. Rocco Totaro, Gianfranco Costantino, Paolo Bellantonio, Caterina Di Carmine, Antonio Carolei, Ciro Mundi, Stefano Ruggieri, and Carmine Marini contributed to analysis and interpretation of the data. Rocco Totaro and Caterina Di Carmine participated in drafting of the paper. Rocco Totaro, Caterina Di Carmine, Gianfranco Costantino, Paolo Bellantonio, Antonio Carolei, Carmine Marini, Aurora Fuiani, and Roberta Fantozzi contributed to critical revision of the paper for important intellectual content. All authors read and approved the final paper.

\section{References}

[1] J. Ingwersen, O. Aktas, P. Kuery, B. Kieseier, A. Boyko, and H.P. Hartung, "Fingolimod in multiple sclerosis: mechanisms of action and clinical efficacy," Clinical Immunology, vol. 142, no. 1, pp. 15-24, 2012.

[2] M. H. Hyland and J. A. Cohen, "Fingolimod," Neurology: Clinical Practice, vol. 1, no. 1, pp. 61-65, 2011.

[3] D. Pelletier and D. A. Hafler, "Fingolimod for multiple sclerosis," The New England Journal of Medicine, vol. 366, no. 4, pp. 339347, 2012.

[4] V. Brinkmann, A. Billich, T. Baumruker et al., "Fingolimod (FTY720): discovery and development of an oral drug to treat multiple sclerosis," Nature Reviews Drug Discovery, vol. 9, no. 11, pp. 883-897, 2010. 
[5] A. Groves, Y. Kihara, and J. Chun, "Fingolimod: direct CNS effects of sphingosine 1-phosphate (S1P) receptor modulation and implications in multiple sclerosis therapy," Journal of the Neurological Sciences, vol. 328, no. 1-2, pp. 9-18, 2013.

[6] R. Deogracias, M. Yazdani, M. P. J. Dekkers et al., "Fingolimod, a sphingosine-1 phosphate receptor modulator, increases BDNF levels and improves symptoms of a mouse model of Rett syndrome," Proceedings of the National Academy of Sciences of the United States of America, vol. 109, no. 35, pp. 14230-14235, 2012.

[7] L. Kappos, E.-W. Radue, P. O'Connor et al., "A placebo-controlled trial of oral fingolimod in relapsing multiple sclerosis," The New England Journal of Medicine, vol. 362, no. 5, pp. 387401, 2010.

[8] E.-W. Radue, P. O’Connor, C. H. Polman et al., "Impact of fingolimod therapy on magnetic resonance imaging outcomes in patients with multiple sclerosis," Archives of Neurology, vol. 69, no. 10, pp. 1259-1269, 2012.

[9] J. A. Cohen, F. Barkhof, G. Comi et al., "Oral fingolimod or intramuscular interferon for relapsing multiple sclerosis," The New England Journal of Medicine, vol. 362, no. 5, pp. 402-415, 2010.

[10] V. Devonshire, E. Havrdova, E. W. Radue et al., "Relapse and disability outcomes in patients with multiple sclerosis treated with fingolimod: subgroup analyses of the doubleblind, randomised, placebo-controlled FREEDOMS study," The Lancet Neurology, vol. 11, no. 5, pp. 420-428, 2012.

[11] A. Huggins and R. C. Sergott, "Gilenya safety update: cardiac and macula," Current Opinion in Ophthalmology, vol. 23, no. 6, pp. 457-459, 2012.

[12] A. Huggins and R. C. Sergott, "Background and rationale for mechanism of action, efficacy, and safety of fingolimod (Gilenya), the first oral therapy for remitting-relapsing multiple sclerosis: with special emphasis upon visual safety," Current Opinion in Ophthalmology, vol. 22, no. 6, pp. 447-450, 2011.

[13] G. Comi, L. Kappos, J. Palace et al., "Cardiac safety of fingolimod $0.5 \mathrm{mg}$ during the first dose observation in 4-month, openlabel, multi-center FIRST study in patients with relapsing MS," Neurology, vol. 78, article S41.003, 2012.

[14] S. Braune, M. Lang, and A. Bergmann, "Second line use of Fingolimod is as effective as Natalizumab in a German outpatient RRMS-cohort," Journal of Neurology, vol. 260, no. 12, pp. 2981-2985, 2013.

[15] M. Cohen, E. Maillart, A. Tourbah et al., "Switching from natalizumab to fingolimod in multiple sclerosis: a French prospective study," JAMA Neurology, vol. 71, no. 4, pp. 436-441, 2014.

[16] C. H. Polman, P. W. O'Connor, E. Havrdova et al., "A randomized, placebo-controlled trial of natalizumab for relapsing multiple sclerosis," The New England Journal of Medicine, vol. 354, no. 9, pp. 899-910, 2006.

[17] R. Totaro, A. Lugaresi, P. Bellantonio et al., "Natalizumab treatment in multiple sclerosis patients: a multicenter experience in clinical practice in Italy," International Journal of Immunopathology and Pharmacology, vol. 27, no. 2, pp. 147-154, 2014.

[18] G. Comi, "Position and practical use of fingolimod in Europe," Clinical and Experimental Neuroimmunology, vol. 5, no. 1, pp. 19-33, 2014. 


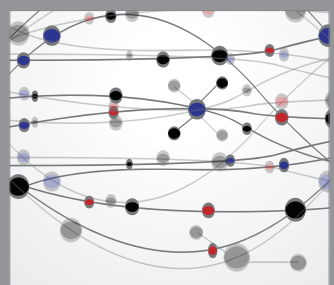

The Scientific World Journal
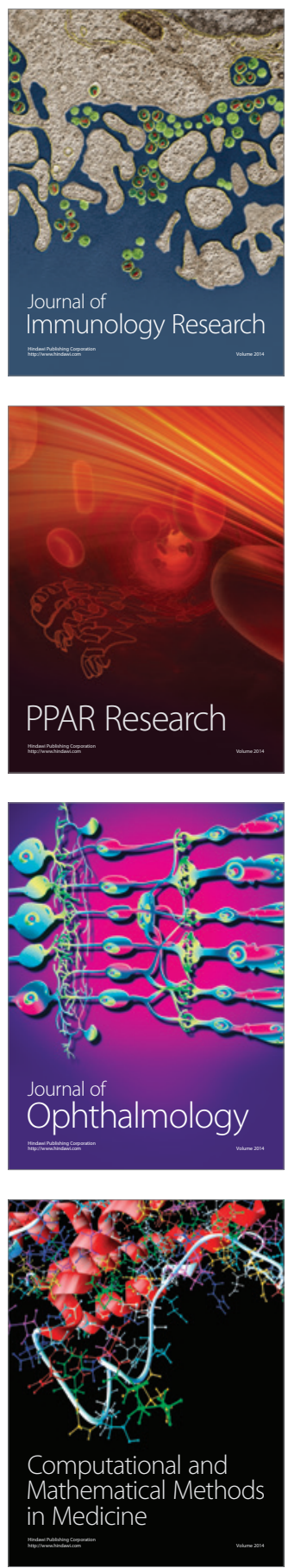

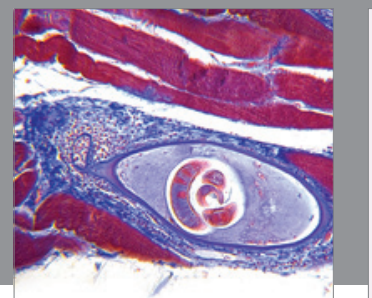

Gastroenterology

Research and Practice
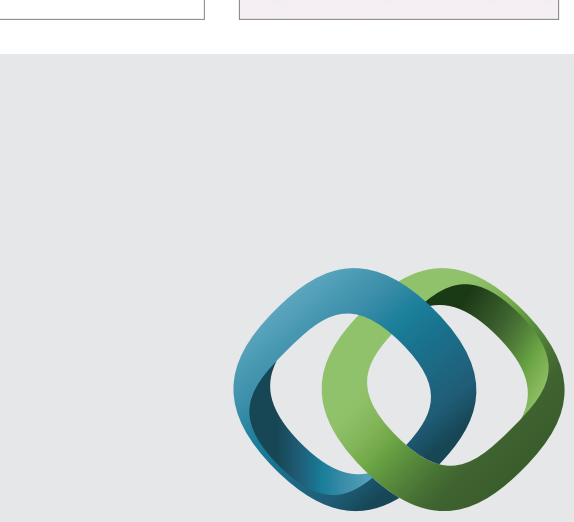

\section{Hindawi}

Submit your manuscripts at

http://www.hindawi.com
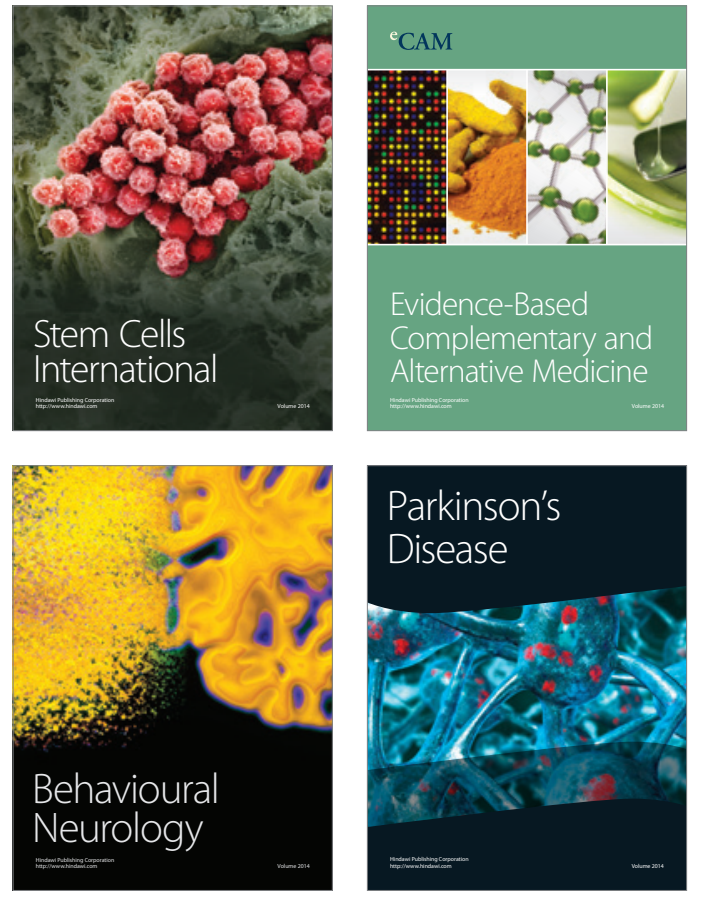
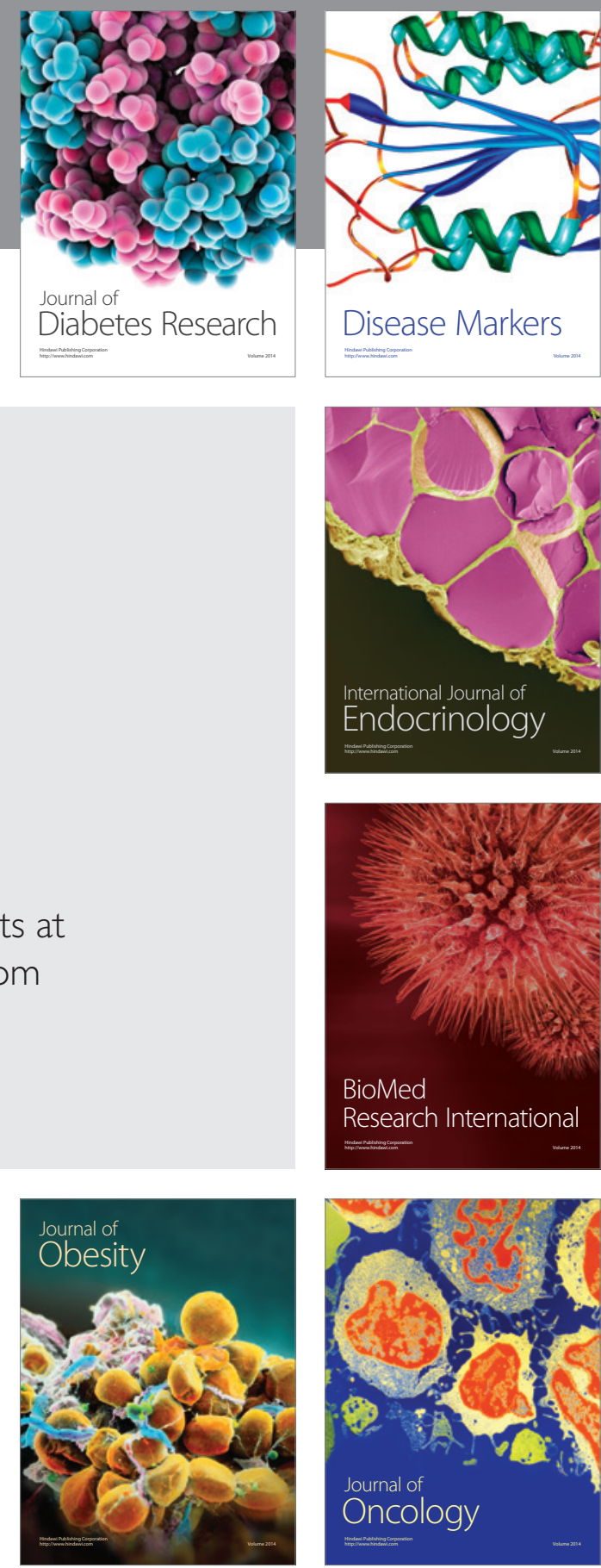

Disease Markers
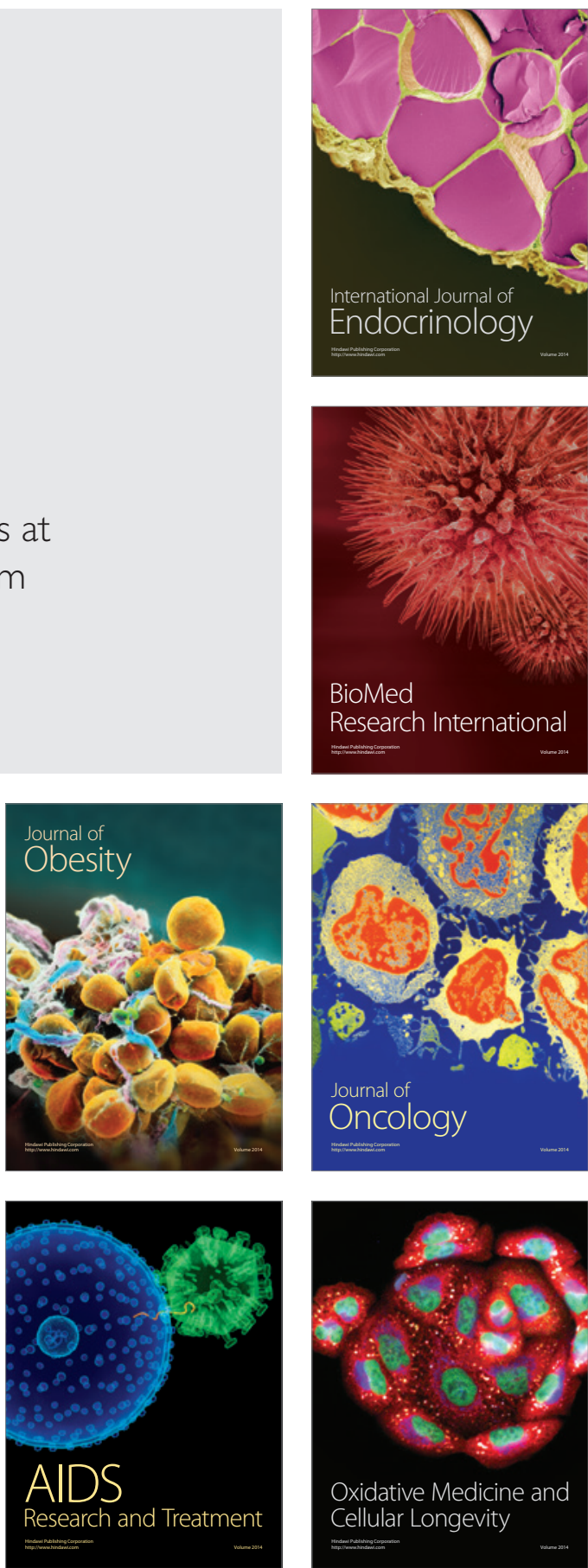